\section{Synchronous cytomegalovirus infection in a newly diagnosed ulcerative colitis patient}

\author{
Jin Yu Chieng, ${ }^{1}$ Yasotha Sugumaran, ${ }^{1}$ \\ Sellymiah Adzman, ${ }^{2}$ Pan Yan ${ }^{3}$ \\ ${ }^{1}$ Department of Medicine, Faculty of \\ Medicine and Health Science, Universiti \\ Putra Malaysia, Selangor; ${ }^{2}$ Department \\ of Pathology, Serdang Hospital,
} Selangor; ${ }^{3}$ Department of Biomedical Science, The University of Nottingham, Semenyih, Malaysia

\begin{abstract}
A 61-year-old Punjabi female patient presented with six months history of mild abdominal discomfort with bloody diarrhea. She did not have underlying chronic medical illness; she neither took steroid nor immunosuppressant. She was found anemic, thrombocytosis, and elevated C-reactive protein. Colonoscopy showed moderate left sided colitis, with histopathology evidence of ulcerative colitis (UC) with cytomegalovirus (CMV) infection. Her serum anti-CMV IgM antibody was detected. She was treated with intravenous ganciclovir, together with 5-ASA and tapering dose of steroid. Anemia was corrected. Subsequent clinic reviews and follow up endoscopies showed dramatically improvement. CMV colitis should be considered for the patients presenting with moderate to severe UC. Early prescription of antiviral would be beneficial in the treatment of flare of UC.
\end{abstract}

\section{Introduction}

Cytomegalovirus is a member of the Herpesviridae family and is a common infection in humans with a prevalence of over $70 \% .^{1}$ Disease acquisition is usually asymptomatic in healthy people, but it leads to lifelong latent infection. Blood serology tests show that 60 to $90 \%$ of adults have had a CMV infection at some time.

Gastrointestinal infections with CMV, especially colitis, are usually found in immunocompromised patients and rarely affect immunocompetent subjects. The association between inflammatory bowel disease especially UC and CMV has been recognized for over half a decade. There are few reports of CMV colitis in immunocompetent hosts, ${ }^{2,3}$ with Blair reported the first case in $1992 .{ }^{4}$
Most of the cases reported as flare of $\mathrm{UC}$ with CMV infection. Here, we report a case of synchronous CMV infection in a patient with newly diagnosed UC. Our purpose is therefore to familiarize clinicians involved with the diagnosis and management of gastroenterological diseases with this entity.

\section{Case Report}

A 61 year old Punjabi female patient presented with six months history of mild abdominal discomfort with bloody diarrhea. She denied of fever, nor weight loss. She did not have underlying chronic medical illness; she neither took steroid nor immunosuppressant. Further questioning, she denied of family history of inflammatory bowel disease or colorectal cancer.

She was pale, with otherwise normal abdominal examination.

Biochemistry showed normochromic normocytic anemia with hemoglobin level $8.8 \mathrm{~g} / \mathrm{dL}$, reactive thrombocytosis; and elevated C-reactive protein. Her stool oval and cyst, and culture both showed negative. Tumor marker CEA was within normal range. Her serum anti-CMV IgM antibody was detected. Colonoscopy showed moderate left sided colitis, with hemorrhagic mucosal erosions at recto-sigmoid region and descending colon (Figure 1), otherwise healthy mucosa of the other parts of the colon till terminal ileum. Few target biopsies taken and the histopathology examination reported as ulcerative colitis (presence of crypt abscesses, cryptitis, crypt distortion, lymphoplasmacytosis, and neutrophil infiltration) (Figure 2) with CMV infection (classical inclusion bodies with nuclear positivities of CMV immunestaining) (Figure 3). Otherwise, no granuloma, dysplasia, or malignancy noted. Tuberculosis had been ruled out as well, proven by the normal skin mantoux test, and clear chest X-ray.

She was warded and blood transfused to keep the haemoglobin level above $10 \mathrm{~g} / \mathrm{dL}$. She was treated with two weeks of intraveous ganciclovir ( $10 \mathrm{mg} / \mathrm{kg} /$ day $)$, together with both oral and suppository 5ASA with intravenous hydrocortisone 100 mg tds initially followed by tapering dose of oral prednisolone over three months. She improved dramatically and discharged well.

Subsequent colonoscopy reviewed mucosal healing (Figure 4). And she is on maintenance dose of oral 5-ASA now.

\section{Discussion}

The association between CMV and IBD
Correspondence: Jin Yu Chieng, Department of Medicine, Faculty of Medicine and Health Science, Putra University of Malaysia, 43400 Serdang UPM, Selangor, Malaysia.

Tel.: +6.016.8580155.

E-mail: cjy511@yahoo.com

Key words: Cytomegalovirus, ulcerative colitis, antiviral.

Contributions: the authors contributed equally.

Conflict of interest: the authors declare no potential conflict of interest.

Received for publication: 13 September 2017 Accepted for publication: 26 October 2017.

This work is licensed under a Creative Commons Attribution NonCommercial 4.0 License (CC BY-NC 4.0).

(C) Copyright J.Y. Chieng et al., 2017

Licensee PAGEPress, Italy

Gastroenterology Insights 2017; 8:6886

doi:10.4081/gi.2017.6886

has remained a topic of ongoing controversy since long ago. In 1961, Powell et al. had described a patient with UC and cytomegalic inclusion disease. ${ }^{5}$

Overall prevalence of CMV is unknown in IBD patients. Most of studies have been carried out using a selected patient group and using different diagnostic methods, so that the available data include a wide range of prevalences of $4.5-70 \%$, which could be biased. Domènech et al. has reported a high prevalence of CMV infection $(70 \%)$ in UC patients in Spain, ${ }^{6}$ which has a similar percentage to that of the general population. Patients with inactive or mild-moderate UC did not show an increased risk of CMV colitis. However, literatures review that the prevalence of CMV infection could be higher in severe or steroid-refractory colitis, with risk factors of female gender, older age, pancolitis and azathioprine treatment. ${ }^{7,8}$ It is not known whether the virus exacerbates the disease or simply appears as a bystander. ${ }^{9}$

Typical endoscopic findings of CMV colitis alone are microerosions, deep ulcers and pseudotumoral lesions. ${ }^{10}$ Most studies in patients with IBD, specifically in active UC, have not found specific endoscopic features. $7,11,12$ Suzuki et al. found that punched-out ulceration and longitudinal ulceration exhibited relatively high sensitivity and specificity for CMV colitis in inflammatory bowel disease. ${ }^{13}$

Histological diagnosis is considered the gold standard for diagnosing CMV disease in the gastrointestinal tract, ${ }^{14,15}$ in which 


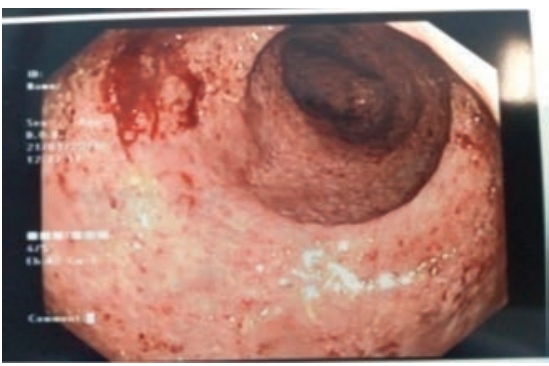

Figure 1. Pre-treatment colonoscopy reviewed of moderate colitis - hemorrhagic mucosal erosions at recto-sigmoid region and descending colon.

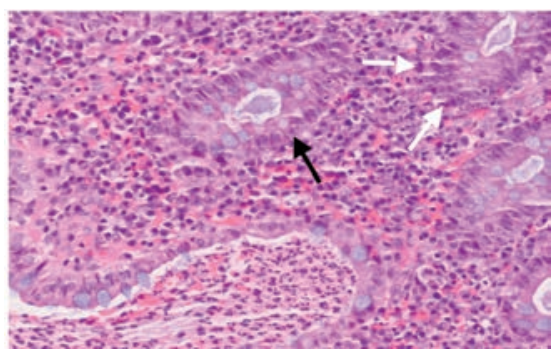

Figure 2. Crypt abscess (black arrow) with cryptitis (white arrow) (H\&E x400).

had been applied in our patient as well.

Most authors recommend the use of antivirals (a full 14 day course of intravenous gancyclovir) in steroid-refractory UC flare-up and CMV positive patients. ${ }^{6,16,17}$ Antiviral treatment can significantly decrease the mortality rate and need for surgery.

\section{Conclusions}

Our report is unique that $\mathrm{CMV}$ infection was found in a newly diagnosed ulcerative colitis patient, which is considered as a synchronous finding. CMV colitis should be considered for all patients presenting with moderate to severe UC, especially in those patients who are resistant to steroids or immunosuppressants. ${ }^{18}$ Early commencement of antiviral would be beneficial in the management of flare of ulcerative colitis.

\section{References}

1. Lockwood MR, Liddle J, Kitsanta P.

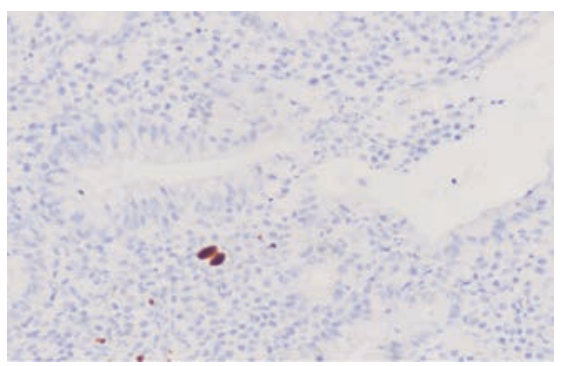

Figure 3. Two nuclear positivities of cytomegalovirus immunostain (H\&E x200).

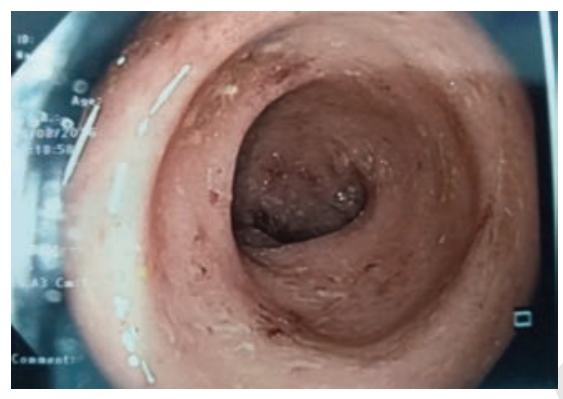

Figure 4. Post-treatment colonoscopy reviewed mucosal healing.

Cytomegalovirus colitis - an unusual cause for diarrhoea in an elderly woman. Age Ageing 2006;35:198-200.

2. Galiatsatos P, Shrier I, Lamoureux E, Szilagyi A. Meta-analysis of outcome of cytomegalovirus colitis in immunocompetent hosts. Dig Dis Sci 2005;50:609-16.

3. Ng FH, Chau TN, Cheung TC, et al. Cytomegalovirus colitis in individuals without apparent cause of immunodeficiency. Dig Dis Sci 1999;44:945-52.

4. Blair SD, Forbes A, Parkins RA. CMV colitis in an immunocompetent adult. J R Soc Med 1992;85:238-9.

5. Powell RD, Warner NE, Levine RS, Kirsner JB. Cytomegalic inclusion disease and ulcerative colitis; report of a case in a young adult. Am J Med 1961;30:334-40.

6. Domènech E, Vega R, Ojanguren I, et al. Cytomegalovirus infection in ulcerative colitis: a prospective, comparative study on prevalence and diagnostic strategy. Inflamm Bowel Dis 2008;14:1373-9.

7. Wada Y, Matsui T, Matake $H$, et al. Intractable ulcerative colitis caused by cytomegalovirus infection: a prospective study on prevalence, diagnosis, and treatment. Dis Colon Rectum 2003;46:S59-65.

8. Kojima T, Watanabe T, Hata K, et al. Cytomegalovirus infection in ulcerative colitis. Scand J Gastroenterol 2006;41: 706-11.

9. Wakefield AJ, Fox JD, Sawyerr AM, et al. Detection of herpesvirus DNA in the large intestine of patients with ulcerative colitis and Crohn's disease using the nested polymerase chain reaction. J Med Virol 1992;38:183-90.

10. Ljungman P, Griffiths P, Paya C. Definitions of cytomegalovirus infection and disease in transplant recipients. Clin Infect Dis 2002;34:1094-7.

11 Sakamoto I, Shirai T, Kamide T, et al. Cytomegalovirus enterocolitis in an immunocompetent individual. J Clin Gastroenterol 2002;34:243-6.

12. Iida $T$, Ikeya $K$, Watanabe $F$, et al. Looking for endoscopic features of cytomegalovirus colitis: a study of 187 patients with active ulcerative colitis, positive and negative for cytomegalovirus. Inflamm Bowel Dis 2013;19:1156-63.

13. Suzuki H, Kato J, Kuriyama M, et al. Specific endoscopic features of ulcerative colitis complicated by cytomegalovirus infection. World J Gastroenterol 2010;16:1245-51.

14. Wu GD, Shintaku IP, Chien K, Geller SA. A comparison of routine light microscopy, immunohistochemistry, and in situ hybridization for the detection of cytomegalovirus in gastrointestinal biopsies. Am J Gastroenterol 1989; 84:1517-20.

15. Kandiel A, Lashner B. Cytomegalovirus colitis complicating inflammatory bowel disease. Am J Gastroenterol 2006;101:2857-65.

16. Christidou A, Zambeli E, Mantzaris G. Cytomegalovirus and inflammatory bowel disease: pathogenicity, diagnosis and treatment. Ann Gastroenterol 2007;20:110-5.

17. Hashash JG, Refaat M, Abdulbaki A, et al. Cytomegalovirus colitis. Clevel Clin J Med 2012;79:12-3.

18. Sager K, Alam S, Bond A, et al. Cytomegalovirus and inflammatory bowel disease. Aliment Pharmacol Ther 2015;41:725-33. 